\title{
Chronic Daily House Dust Mite Exposure in Mice is an Effective Model to Quantify the Effect of Pharmacologic Agents on Discrete Stages of Artery Remodeling in Pulmonary Hypertension

\author{
Lea C. Steffes and Maya E. Kumar*
}

Department of Pediatrics, Division of Pulmonary Medicine, Stanford University School of Medicine, Palo Alto, CA, USA

*For correspondence: mayak@stanford.edu

\begin{abstract}
[Abstract] Pulmonary hypertension $(\mathrm{PH})$ is a heterogenous and incurable disease marked by varying degrees of pulmonary vascular remodeling. This vascular remodeling, which includes thickening of the smooth muscle layer (an early finding) and formation of occlusive neointimal lesions (a late finding) in the pulmonary arteries, is a major driver of morbidity and mortality in $\mathrm{PH}$. Available $\mathrm{PH}$ therapies consist of vasodilators that do not specifically target lesion formation or expansion and neither prevent progression nor reverse disease. This paucity of curative treatments highlights the need for new drug discovery targeting crucial steps of artery remodeling in $\mathrm{PH}$. The cell dynamics and molecular signals driving neointimal lesion formation have been difficult to elucidate as classic mouse models of $\mathrm{PH}$ do not develop neointima. Here, we detail the methods to generate a robust and non-genetic mouse model of $\mathrm{PH}$ with medial thickening and neointimal lesion formation in the pulmonary arteries, through chronic exposure to an inflammatory stimulus-house dust mite (HDM). This model rapidly generates humanlike pulmonary arterial lesions following a reproducible time course, allowing scrutiny of the cellular and molecular mechanisms controlling each stage of artery remodeling. Further, we outline optimal tissue handling, sectioning, and staining methodologies for detailed quantitative analysis of artery medial thickening and neointimal lesion formation and expansion. Finally, we present a method for staged pharmacologic intervention to identify molecules and pathways required at each step of the pulmonary arterial remodeling process. The advantages of this mouse model of $\mathrm{PH}$ over currently available animal models are five-fold. (i) It allows the use of the full range of genetic and single cell tools available in mice to manipulate and study the process of vascular remodeling seen in human disease, including the formation of neointimal lesions in a controlled and cell specific manner. (ii) The vascular lesions develop in a stereotyped manner with predictable timing, allowing for pharmacologic manipulation at discrete stages of vessel remodeling. (iii) It is rapid, with development of $\mathrm{PH}$ and vascular remodeling in a timeframe of two to eight weeks. (iv) It uses simple techniques and requires neither surgery, unusual equipment, or extensive personnel training. (v) The staining and quantitation methodologies we present are a significant improvement over those currently in use in the field. We hope that dissemination of this model and the associated detailed methods will speed up the development of novel and more effective $\mathrm{PH}$ therapeutics.
\end{abstract}


Please cite this article as: Steffes, L. C. and Kumar, M. E. (2022). Chronic Daily House Dust Mite Exposure in Mice is an Effective Model to Quantify the Effect of Pharmacologic Agents on Discrete Stages of Artery Remodeling in Pulmonary Hypertension. Bio-protocol 12(01): e4273. DOI:

\section{Graphic abstract:}

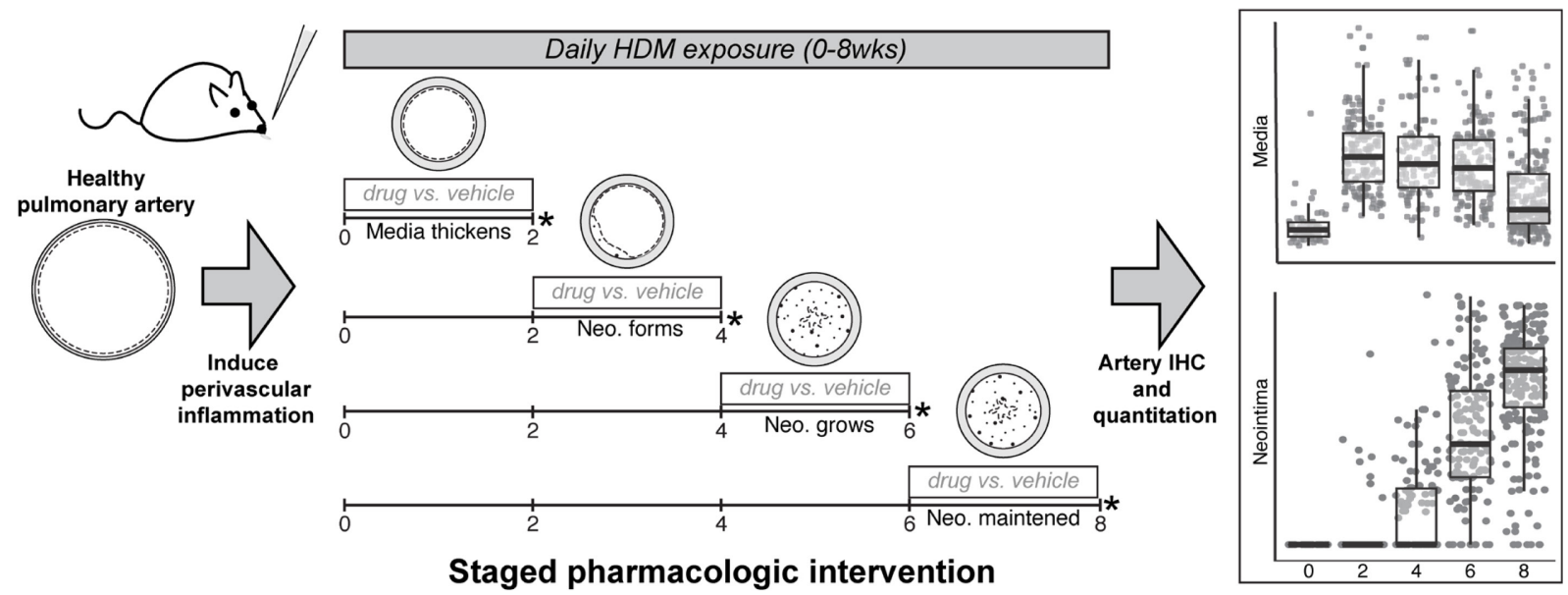

Chronic perivascular inflammation induces medial thickening and neointima formation in pulmonary arteries, following a stereotyped time course, and allowing staged pharmacologic intervention during specific remodeling events, as well as quantitative assessment of vascular changes.

Keywords: Pulmonary hypertension, Neointima, Neointimal lesion, Mouse model, Lung perfusion, Lung inflation, Elastin, Vessel quantitation, House dust mite, Perivascular inflammation, Staged pharmacology

[Background] Pulmonary hypertension $(\mathrm{PH})$ is a fatal disease affecting children and adults with no available cure. It affects patients of all ages and is estimated to result in 200,000 hospitalizations and 15,000 deaths each year (Lau et al., 2017). PH encompasses a heterogenous group of pathologic processes that result in elevation in pulmonary arterial pressures (Simonneau et al., 2019). In all forms of $\mathrm{PH}$, there is prominent vascular remodeling of the arteries of the pulmonary circulation, with thickening and contraction of the medial smooth muscle layer followed by the formation of occlusive 'neointimal lesions' in medium and small pulmonary arteries. These pathological changes narrow vessel lumens (Figure 1), increase pulmonary vascular resistance, and ultimately result in right heart failure and death (Humbert et al., 2018).

Available $\mathrm{PH}$ therapies consist of pulmonary vasodilators which do not specifically target artery remodeling and do not prevent progression or reverse disease (Spiekerkoetter et al., 2019). Current mouse models either reproduce only limited features of the disease (Stenmark et al., 2006), or are driven by multi-allelic genetic approaches (Dai et al., 2016) that limit the use of additional genetic tools to study disease processes (Gomez-Arroyo et al., 2012). A non-genetically driven murine model of pulmonary hypertension, with robust pulmonary vascular changes, should allow a granular analysis of the cellular and molecular basis of artery remodeling in $\mathrm{PH}$, and could facilitate the development of new more effective therapies.

Here, we present a detailed protocol for inducing $\mathrm{PH}$ in mice that reproduces human vasculopathy in a tractable and reproducible time frame, with simple non-genetic methods. The vascular changes 
Please cite this article as: Steffes, L. C. and Kumar, M. E. (2022). Chronic Daily House Dust Mite Exposure in Mice is an Effective Model to Quantify the Effect of Pharmacologic Agents on Discrete Stages of Artery Remodeling in Pulmonary Hypertension. Bio-protocol 12(01): e4273. DOI:

observed in this model closely mimic those observed in $\mathrm{PH}$ patients, and include medial thickening, formation and expansion of occlusive neointimal lesions, elaboration of the bronchial circulation, and muscularization of the veins (Steffes et al., 2020). Most notably, pulmonary artery remodeling in this model occurs in three temporally distinct steps, beginning with medial thickening, followed by establishment of small neointimal lesions, and finally rapid proliferation of neointimal cells to occlude the lumen, shown in Figure 2A-2B. These stages occur during well-defined temporal windows, providing a tractable framework through which to quantify the effect of pharmacologic or genetic manipulation during discrete events in artery remodeling. Additionally, we provide tissue preparation, staining, and quantification protocols to visualize key elements of arterial lesions with multicolor fluorescence microscopy highlighting elastin, smooth muscle, neointima, and endothelium.

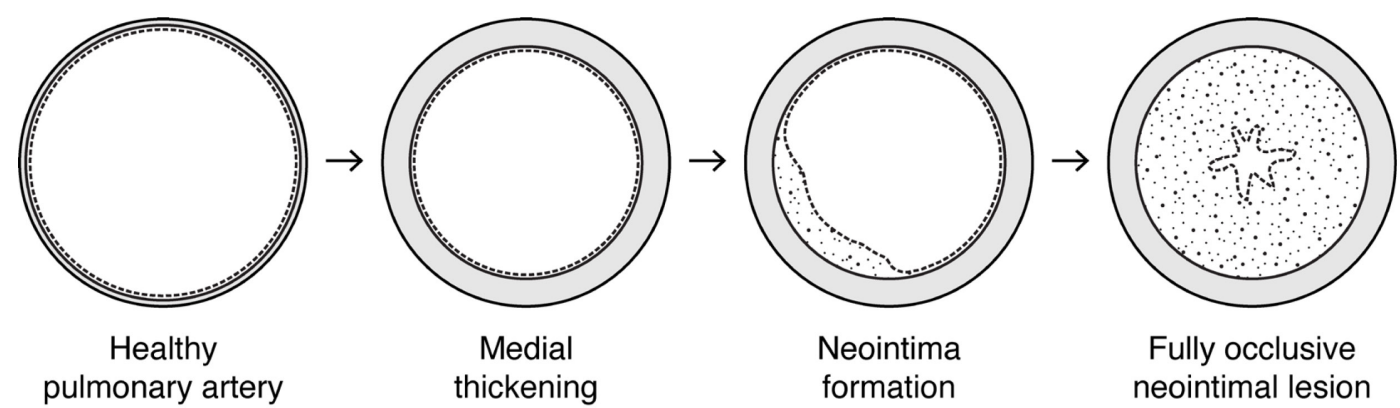

Figure 1. Progressive artery remodeling in human pulmonary arterial hypertension (PAH).

Pulmonary arterial hypertension is marked by progressive remodeling of small to medium sized pulmonary arteries and arterioles. Healthy arteries have a thin medial smooth muscle cell layer (grey) located between the internal and external elastic laminae (black solid lines) and a lumen lined with endothelium (dashed line). In early PAH and other forms of PH there is thickening of the medial smooth muscle cell layer. Later in disease, neointimal lesions (white speckled area) form between the internal elastic lamina and the endothelium and grow to occlude the vessel lumen in end stage disease. 
Please cite this article as: Steffes, L. C. and Kumar, M. E. (2022). Chronic Daily House Dust Mite Exposure in Mice is an Effective Model to Quantify the Effect of Pharmacologic Agents on Discrete Stages of Artery Remodeling in Pulmonary Hypertension. Bio-protocol 12(01): e4273. DOI:
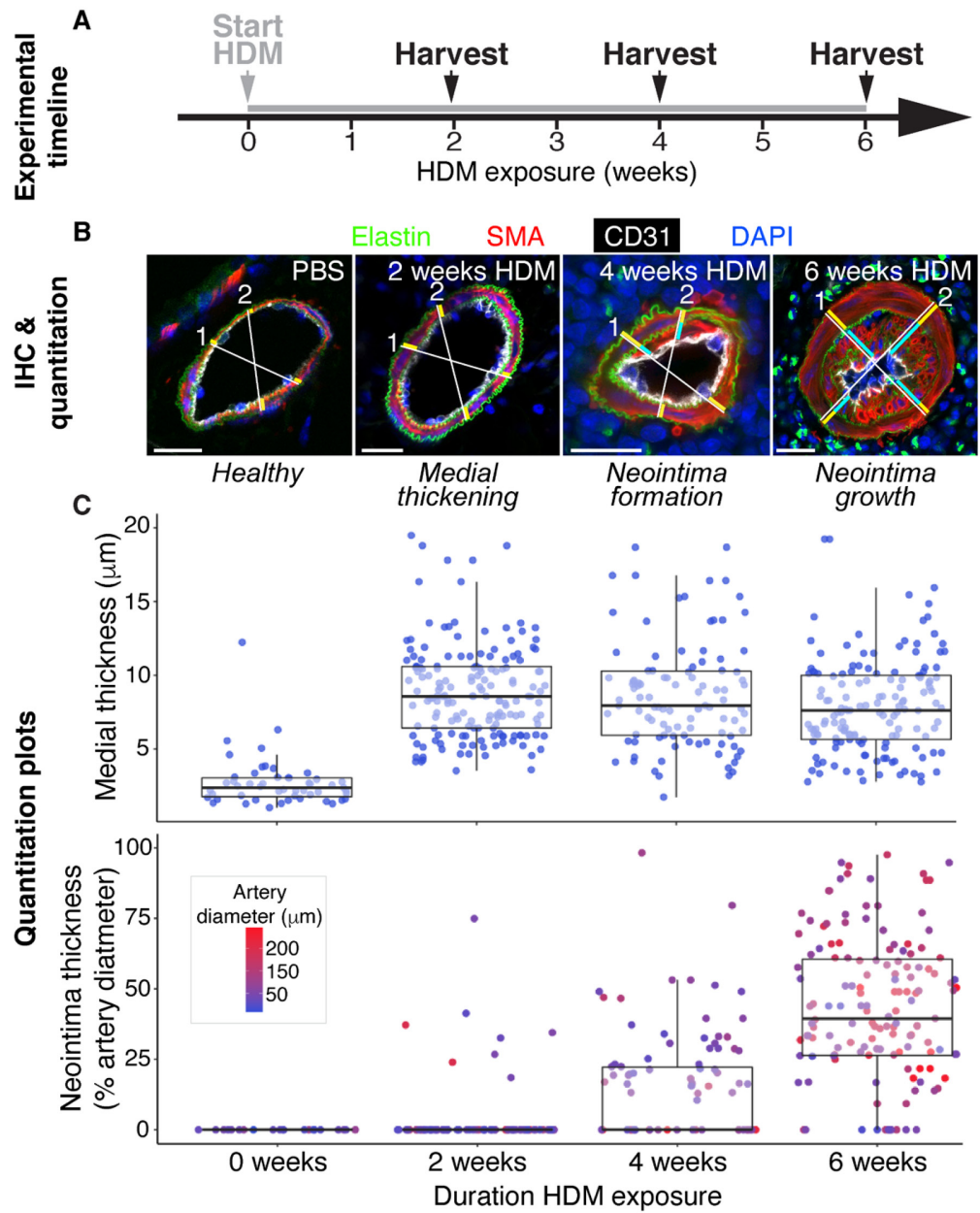

Figure 2. Protocol overview summarizing experimental timeline, immunohistochemistry (IHC), and vessel remodeling quantification.

A-B. Daily intranasal HDM exposure in mice induces discrete stages of pulmonary arterial remodeling over 6 weeks, with progression from healthy arteries prior to HDM administration, to medial thickening seen in animals harvested at 2 weeks, neointimal formation after 4 weeks, and diffuse neointimal lesion growth and luminal occlusion by 6 weeks. B. Confocal micrographs of IHC performed on $20 \mu \mathrm{m}$ cryosections with the optimized staining protocol outlined in Table 1 allow detailed quantification of artery remodeling, by clearly visualizing the internal and external elastic laminae (elastin/hydrazide, green), the medial smooth muscle cell layer [smooth muscle actin (SMA)+ cells, red, between the internal and external elastic laminae], the endothelium (CD31, white), neointimal lesions (SMA+ cells between the internal elastic lamina and the endothelium), and nuclei (DAPI, blue). To quantitate degree of vessel remodeling, two orthogonal measurement axes are drawn per vessel, and their length in micrometer are recorded and represent external vessel diameter (white lines), medial thickness (yellow bars), and neointimal thickness, when present (light blue bars). C. Vessel quantitation measurements for $\sim 50$ vessels per timepoint are displayed in scatterplots, with values grouped by duration of HDM exposure. Absolute medial thickness (top plot) and neointimal thickness expressed as percentage of vessel diameter (bottom plot) are useful ways to visualize the data. The media thickens during the first two weeks of HDM exposure, and remains thickened through the subsequent 4 weeks of HDM exposure. 
Please cite this article as: Steffes, L. C. and Kumar, M. E. (2022). Chronic Daily House Dust Mite Exposure in Mice is an Effective Model to Quantify the Effect of Pharmacologic Agents on Discrete Stages of Artery Remodeling in Pulmonary Hypertension. Bio-protocol 12(01): e4273. DOI:

Neointima is established after 4 weeks HDM exposure, and undergoes rapid expansion during weeks 4-6. Each dot represents a discrete vessel measurement. Dot color in the bottom plot reports vessel diameter. Scale bars in B are $20 \mu \mathrm{m}$.

\section{Materials and Reagents}

A. Animals

Eight to ten-week-old BALB/c female mice (Charles River, strain 028)

B. House dust mite preparation and administration

1. $1.5 \mathrm{~mL}$ Eppendorf polypropylene tube with locking cap (Fisher Scientific, catalog number: 05402-25)

2. $200 \mu \mathrm{L}$ pipette (Millipore Sigma, Eppendorf, catalog number: Z740441)

3. $200 \mu \mathrm{L}$ pipette filter tips (Fisher Scientific, catalog number: 05-413-952)

4. Isoflurane (VetOne, catalog number: 502017)

C. Lung dissection, perfusion, and inflation

1. $70 \%$ ethanol (Fisher Scientific, catalog number: NC9663244)

2. Spray bottle (Fisher Scientific, catalog number: 02-991-721)

3. $50 \mathrm{~mL}$ syringe (Fisher Scientific, catalog number: 14-817-57)

4. Butterfly needle, $27 \mathrm{G}$ (Fisher Scientific, catalog number: 02-664)

5. Aluminum foil sheets (Fisher Scientific, catalog number: 01-213-106)

6. Styrofoam pad (e.g., lid of Styrofoam box, to use as dissection pad)

7. $21 \mathrm{G} 1$ " needles (to use as pins) (Fisher Scientific, catalog number: 14-840-92)

8. C-fold paper towels

9. Silk surgical suture thread (Fine Science Tools, catalog number: 18020-01)

10. $5 \mathrm{~mL}$ syringe (Fisher Scientific, catalog number: 14-829-45)

11. 20 G 0.5" blunt needle/cannula (Sai Infusion, catalog number: B20-50)

12. Falcon $50 \mathrm{~mL}$ conical tubes (Fisher Scientific, catalog number: 14-432-22)

13. Falcon $15 \mathrm{~mL}$ conical tubes (Fisher Scientific, catalog number: 14-959-53A)

14. Ice

15. $90 \mathrm{~mm}$ Petri dishes (Fisher Scientific, catalog number: 07-202-028)

16. Water bath (Fisher Scientific, catalog number: 07-202-155)

17. $10 \%$ neutral buffered formalin (Fisher Scientific, catalog number: 22-026-213)

18. Lab marker (Fisher Scientific, catalog number: 13-379-4)

D. Tissue Handling and Staining

1. $90 \mathrm{~mm}$ Petri dishes (Fisher Scientific, catalog number: 07-202-028)

2. $7.5 \mathrm{~mL}$ transfer pipettes (Fisher, catalog number: 13-711-47) 
Please cite this article as: Steffes, L. C. and Kumar, M. E. (2022). Chronic Daily House Dust Mite Exposure in Mice is an Effective Model to Quantify the Effect of Pharmacologic Agents on Discrete Stages of Artery Remodeling in Pulmonary Hypertension. Bio-protocol 12(01): e4273. DOI:

3. Falcon $15 \mathrm{~mL}$ conical tubes (Fisher Scientific, catalog number: $14-959-53 \mathrm{~A}$ )

4. Optimal cutting temperature (OCT) compound (Sakura, catalog number: 4583)

5. $25 \mathrm{~mm} \times 20 \mathrm{~mm} \times 5 \mathrm{~mm}$ specimen cryomolds (Fisher Scientfic, catalog number: NC9643511)

6. Biopsy tissue cassette (Sakura, catalog number: 4154-01)

7. Methanol wash bottle $(\times 4)$ (Fisher Scientific, catalog number: 03-409-11H)

8. Superfrost+ microscope slides (25/75/1 mm) (Fisher Scientific, catalog number: $12-550-15)$

9. Glass cover slips (24×60-1) (Fisher Scientific, catalog number: $12-545-\mathrm{MP}$ )

10. Slide storage boxes (Fisher Scientific, catalog number: 14-372-86)

11. ImmEdge hydrophobic pen (Vector Labs, catalog number: $\mathrm{H}-4000$ )

12. $1.5 \mathrm{~mL}$ Eppendorf polypropylene tube with locking cap (Fisher Scientific, catalog number: 05402-25)

13. $241 \times 241 \times 20 \mathrm{~mm}$ bioassay dish (Thermo Scientific, catalog number: 240845 ) with four $5 \mathrm{~mL}$ pipettes (Thermo Scientific, catalog number: $170373 \mathrm{~N}$ ) trimmed to size to fit horizontally across the interior of the dish, with two sets of two pipettes placed $50 \mathrm{~mm}$ apart and scotch taped in place as slide supports, and lined with moistened paper towels (shown in Figure 7C). Cover with aluminum foil for dark incubations.

14. Aluminum foil sheets (Fisher Scientific, catalog number: 01-213-106)

15. Antibodies and dyes (detailed in Table 1)

DAPI (cell nuclei) (Invitrogen, catalog number: D1306)

Hydrazide A488 (elastin) (Invitrogen, catalog number: A10436)

Mouse a-SMA Cy3 (SMCs \& neointima) (Sigma, catalog number: C6198)

Armenian Hamster $\alpha-C D 31$ (endothelium) (Bio-Rad, catalog number: MCA1370Z)

Goat a-Armenian Hamster A647 (Invitrogen, catalog number: A-21451)

16. Prolong Gold firm setting mounting medium (Thermo Fisher, catalog number: P36930)

\section{Solutions}

1. 10x Phosphate buffered saline (PBS), diluted to $1 \times \mathrm{PBS}$ in distilled water $\left(\mathrm{dH}_{2} \mathrm{O}\right)$ (Quality Biological, catalog number: 119-069-101). Store at room temperature (RT), and use within 12 months (see Recipes)

2. House dust mite (HDM) (Stallergenes Greer, catalog number: XPB70D3A25). Store at $-20^{\circ} \mathrm{C}$ in $120 \mu \mathrm{L}$ aliquots in PBS, and use within 12 months (see Recipes)

3. Heparin $/ \mathrm{NaCl}$ in PBS. Store at $4^{\circ} \mathrm{C}$, and use within 3 months (see Recipes) $\mathrm{NaCl}$ (Millipore Sigma catalog number: S9888-500G)

Heparin (Millipore Sigma, catalog number: H3393)

4. $2 \%$ low melting point (LMP) agarose in PBS (Fisher Scientific, catalog number: 16-520-050). Store at $4^{\circ} \mathrm{C}$, and use within 3 months (see Recipes)

5. 16\% paraformaldehyde (PFA), diluted to $4 \%$ PFA in PBS (Electron Microscopy Sciences, catalog number: 15170. Store 16\% PFA at RT, and use within 12 months. Keep 4\% PFA solution on ice, and use the same day (see Recipes) 
Please cite this article as: Steffes, L. C. and Kumar, M. E. (2022). Chronic Daily House Dust Mite Exposure in Mice is an Effective Model to Quantify the Effect of Pharmacologic Agents on Discrete Stages of Artery Remodeling in Pulmonary Hypertension. Bio-protocol 12(01): e4273. DOI:

6. $30 \%$ sucrose in PBS (Milipore Sigma, catalog number: S0389). Store at $4^{\circ} \mathrm{C}$, and use within 1 month (see Recipes)

7. Methanol series, diluted with $\mathrm{dH}_{2} \mathrm{O}$ to the desired concentrations (Honeywell, catalog number: BB233-4). Store dilutions in wash bottles at RT, and use within 1 month (see Recipes)

8. PBST (0.1\% Tween 20 in PBS) (Millipore Sigma, catalog number: 9005-64-5). Store at RT, and use within 1 month (see Recipes)

9. Preblock. Store at $4^{\circ} \mathrm{C}$, and use within $24 \mathrm{~h}$ (see Recipes)

Triton X-100 (Millipore Sigma, catalog number: T8787)

Goat serum (Bio-Rad, catalog number: C07SA)

BSA (Millipore Sigma, catalog number: A2153)

\section{Equipment}

A. House dust mite preparation and administration

1. Anesthesia induction chamber (Harvard Apparatus, catalog number: 75-2029)

2. Air filter cannister (Harvard Apparatus, catalog number: 60-0979)

3. Oxygen tank, E 25cf (Praxair, catalog number: OX 2.6MC-E)

4. Isoflurane anesthesia machine (Fisher Scientific, catalog number: 50-154-0189)

B. Lung dissection, perfusion, and inflation

1. Blunt-tip curved serrated iris forceps (Fine Science Tools, catalog number: 11370-31)

2. Pointed-tip curved serrated forceps (Fine Science Tools, catalog number: 11270-20)

3. Dissection scissors (Fine Science Tools, catalog number: 14558-11)

C. Tissue Handling and Staining

1. Blunt-tip curved serrated iris forceps (Fine Science Tools, catalog number: 11370-31)

2. Dissection scissors (Fine Science Tools, catalog number: 14558-11)

3. Cryostat (Leica, model: CM3050S)

4. Vibratome (Leica, model: VT1000 S)

5. Humid chamber (homemade: assembly instructions above in "Materials and Reagents", part D "Tissue Handling and Staining", point 13, and in Figure 7C)

\section{Software}

1. Zen Blue or Zen Black (Carl Zeiss Microscopy)

2. Fij/lmageJ (Imagej.net)

3. Google Sheets (google.com)

4. Excel (Microsoft)

5. R with R Studio (R-project.org; rstudio.com) 


\section{Ggplot2 (ggplot2.tidyverse.org)}

\section{Procedure}

\section{A. Animals}

The standard HDM vascular remodeling time course was generated using eight to ten-week-old BALB/c female mice (Charles River, strain 028). This protocol has been tested and validated in both adult male and adult female mice (>10 weeks old) of multiple genotypes, ages, and body weights, and with multiple lab personnel. No consistent differences in the degree or rate of vascular remodeling in each case have been noted. Before initiating a large-scale experiment, we recommend confirming the timeline of artery remodeling within a lab's particular experimental set up (using mice of the same genetic background, same lab personnel, same HDM lot, etc. that will be used in the larger experiment) with a small number of animals (2-3 per end point). Production of an artery remodeling time course (as shown in Figure $2 \mathrm{~B}$ ) and medial thickness and neointimal lesion quantitation plots at each time point (as shown in Figure $2 \mathrm{C}$ ) will allow for precise staging of windows of vascular remodeling, tailored for each lab's particular conditions and experimental questions.

B. House Dust Mite Preparation and Intranasal Administration

1. HDM preparation

a. Dilute lyophilized house dust mite extract from Stallergenes Greer (XPB70D3A25) with sterile PBS, to $50 \mu \mathrm{g}$ Derp1 protein per $120 \mu \mathrm{L}$, for $4 \times$ stock concentration.

b. Divide HDM stock into $120 \mu \mathrm{L}$ aliquots in $1.5 \mathrm{~mL}$ Eppendorf tubes with locking caps, and store at $-20^{\circ} \mathrm{C}$.

c. Thaw aliquots on day of planned use, and add $350 \mu \mathrm{L}$ of sterile PBS to reach the final $470 \mu \mathrm{L}$ working solution, which will be sufficient for 9 doses. Tips:

i) After stock is thawed and diluted, do not refreeze aliquot. Excess dust mite solution can be stored at $4^{\circ} \mathrm{C}$ and used for up to $48 \mathrm{~h}$.

ii) Because HDM lots vary in both Derp1 concentration and overall protein concentration, preparing sufficient HDM stock for the entire experiment from a single lot is recommended.

2. HDM intranasal administration

a. Anesthetize animals with isoflurane inhalation ( $2.5 \%$ isoflurane nebulized in $2 \mathrm{~L}$ per minute $100 \%$ oxygen) in an anesthesia induction chamber. Tips:

i) Up to 5 animals can be anesthetized at a time.

ii) Animals are sufficiently anesthetized when they no longer react when the induction chamber is gently tipped on its side, but maintain a near normal respiratory rate, 
Please cite this article as: Steffes, L. C. and Kumar, M. E. (2022). Chronic Daily House Dust Mite Exposure in Mice is an Effective Model to Quantify the Effect of Pharmacologic Agents on Discrete Stages of Artery Remodeling in Pulmonary Hypertension. Bio-protocol 12(01): e4273. DOI:

approximately $45 \mathrm{~s}$ in isoflurane. Avoid heavy or prolonged anesthesia, as this will suppress breathing, and impair HDM inhalation.

iii) Because this protocol involves daily anesthesia for a period of weeks, researchers may wish to include both the suggested "no HDM" controls (see step g, below, where an equal volume of sterile PBS is administered, following the same frequency and anesthesia regimen as in HDM-exposed animals) and additional "no anesthesia" controls (where animals are never anesthetized and receive neither PBS nor HDM). For all parameters we have assessed, including RVSP and artery remodeling, we have detected no differences between "no HDM" and "no anesthesia" controls, suggesting repeated anesthesia is not a factor in either of those processes.

b. Draw up $50 \mu \mathrm{L}$ of HDM working solution ( $5 \mu \mathrm{g}$ Derp1 protein) in a $200 \mu \mathrm{L}$ pipette fitted with a fresh disposable tip.

c. Remove one mouse from the anesthesia chamber, scruff the loose fur behind its neck, and position the mouse with its nose up and its belly facing the researcher.

d. Apply individual droplets (each approximately $10 \mu \mathrm{L}$ in volume) of HDM working solution to the surface of one nostril allowing the sedated mouse to breath in each droplet prior to administration of next droplet, shown in Figure 3A-3B.

Tip: Avoid applying large droplets or placing droplet centrally on the mouse's nose (rather than over an individual right or left nostril) to prevent the solution draining into the mouth via the philtrum, as shown in Figure 3C.

e. After $50 \mu \mathrm{L}$ of HDM working solution has been administered, place the anesthetized mouse on its back in its home cage, and allow it to wake from anesthesia. Animals should be moving about the cage normally within 20-30 s.

f. Repeat for all animals daily for 5 consecutive days, allowing a two-day break each week.

g. Administer $50 \mu \mathrm{L}$ of sterile PBS via $200 \mu \mathrm{L}$ pipette with a disposable tip to "no HDM" control mice in the same manner, and following the same schedule as HDM animals.

h. After all animals in a cage are awake and behaving normally, the cage is returned to the rack.

Tip: Dose animals five days per week (for example, Monday through Friday) at approximately the same time each day for 2 to 8 weeks, depending on the stage of vascular remodeling desired (Figure 2B). It is important to allow for a two-day break (for example, Saturday and Sunday), as mice dosed for more than 5 consecutive days can develop outward signs of illness, most commonly a ruffled coat and lethargy. Animals dosed following a "5 days on, 2 days off" schedule, show no outward signs of illness, and behave normally. Throughout this protocol, a "week" of HDM exposure refers to this pattern of five days of daily exposure, followed by 2 days of no exposure, repeated for the indicated number of weeks. 
Please cite this article as: Steffes, L. C. and Kumar, M. E. (2022). Chronic Daily House Dust Mite Exposure in Mice is an Effective Model to Quantify the Effect of Pharmacologic Agents on Discrete Stages of Artery Remodeling in Pulmonary Hypertension. Bio-protocol 12(01): e4273. DOI: 10.21769/BioProtoc.4273.

$\begin{array}{lll}\text { Bio-protocol 12(01): e4273. } & & \text { Bww.bio-protocol.org/e4273 } \\ \text { Dol:10.21769/BioProtoc.4273 }\end{array}$

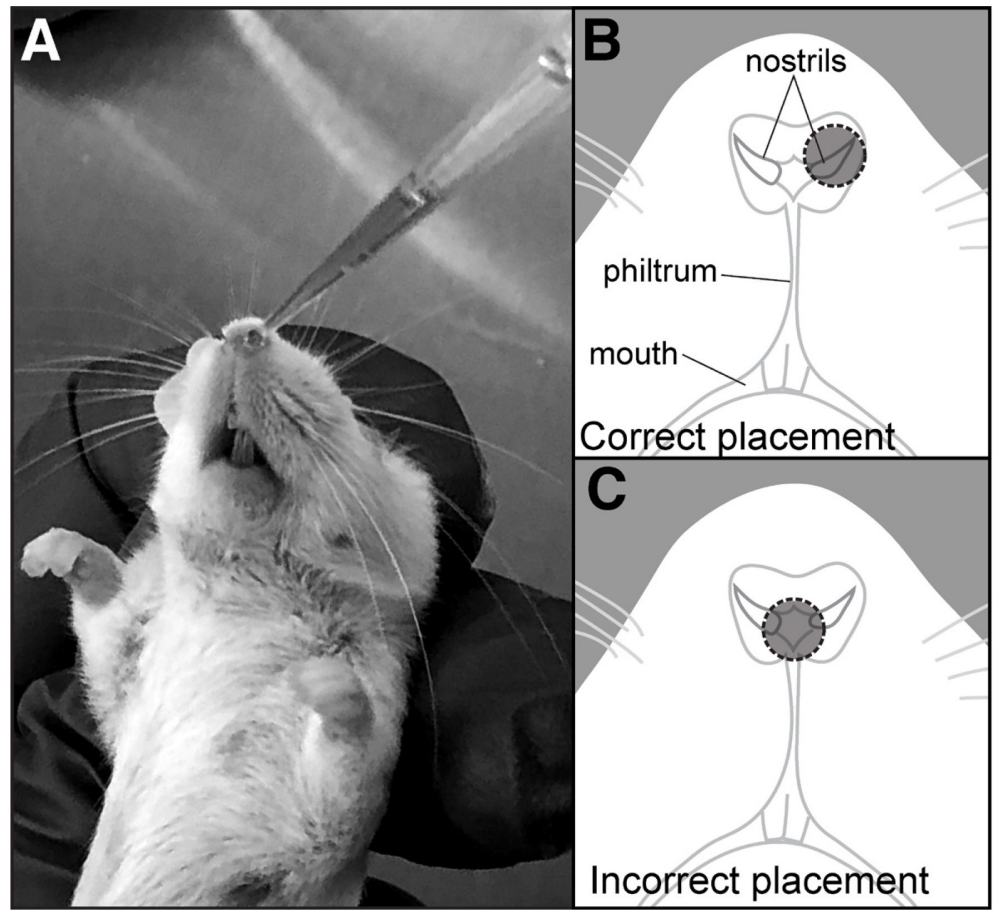

Figure 3. Intranasal administration of HDM extract dissolved in PBS.

HDM solution droplets are placed on the nostrils of isoflurane-anesthetized mice (A-B). Avoid placing the droplets centrally on the nose $(C)$ as solution may drain into the mouth via the philtrum.

\section{Staged Pharmacologic Modulation}

The precise and reproducible timing of artery remodeling seen in this mouse model of $\mathrm{PH}$ permits staged pharmacologic modulation at discrete stages of arterial vascular remodeling: medial thickening (0-2 weeks HDM), neointimal establishment (2-4 weeks HDM), neointimal expansion (46 weeks HDM), and testing of neointimal regression (6-8 weeks HDM), as shown in the schematic in Figure 4. Though the precise experimental design of staged pharmacologic trials will depend on the drug in question, the mode of drug delivery, the drug pharmacokinetics, and which remodeling stages are to be examined in the experiment, the following general guidelines should assist in designing a trial.

1. Administer daily (5 days on 2 days off) intranasal HDM (or PBS) throughout the period of drug or vehicle administration.

2. Experimental animals: Dissolve desired drug in appropriate vehicle solution and dose at suitable intervals based on drug specific pharmacokinetics during the 2-week remodeling window for which the researchers are querying the effect of the drug.

Control animals: Dose with vehicle alone following the same administration technique and schedule as experimental animals.

Tip: Multiple modes of drug delivery can be used with this staged pharmacologic approach, and the optimal mode of drug delivery (intravenous injection, intraperitoneal injection, osmotic pump 
Please cite this article as: Steffes, L. C. and Kumar, M. E. (2022). Chronic Daily House Dust Mite Exposure in Mice is an Effective Model to Quantify the Effect of Pharmacologic Agents on Discrete Stages of Artery Remodeling in Pulmonary Hypertension. Bio-protocol 12(01): e4273. DOI:

infusion, oral gavage, treated water) and the frequency of administration must be determined for each compound, ideally based on prior in vivo studies in mice.

3. Harvest animals on day of completion of the two weeks drug/vehicle exposure window.

4. Complete hemodynamic measurements, tissue collection, and vessel remodeling quantitation (detailed protocols below). This staged pharmacologic modulation allows quantitative comparison of remodeling parameters between drug-treated animals and vehicle controls.
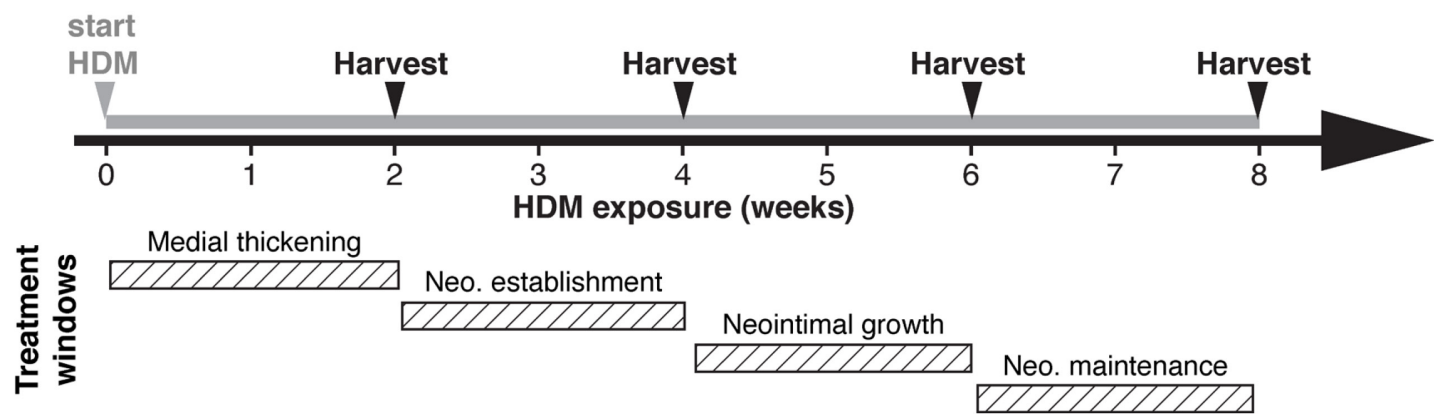

Figure 4. Staged drug treatment during discrete windows of pulmonary arterial remodeling allows quantitative comparison of drug effect on specific stages of artery remodeling.

Specific molecules or pathways of interest can be modulated with administrable agents (e.g., small molecule drugs, antibodies, viral vectors, tamoxifen or tetracycline to alter gene function) during each of the stages of artery remodeling defined in Figure 2, through appropriate timing of drug administration ("Treatment windows"). Comparing drug vs. vehicle treatment groups at each time point, using the quantitation methodology presented in Figure 2, allows identification of a drug's effect on precise steps in the artery remodeling process.

D. Hemodynamic Assessment of Pulmonary Hypertension

Immediately prior to harvest, mice are evaluated for hemodynamic evidence of pulmonary hypertension through right heart catherization and measurement of right ventricular systolic pressure (RVSP), under isoflurane anesthesia following standard protocols (Ma et al., 2016). In the HDM model, significant increase in RVSP is noted after 2 weeks of HDM administration, and this elevation is maintained through 8 weeks of HDM administration (Steffes et al., 2020).

E. Tissue Collection and Handling

1. Preparation for tissue collection

a. Place a water bath near the dissection station and preheat it to $45^{\circ} \mathrm{C}$.

b. Melt prepared $2 \%$ LMP agarose solution in a microwave, swirling often until agarose particles are fully dissolved and the solution is clear.

Tip: Be careful not to concentrate the solution by overboiling.

c. Equilibrate $2 \%$ LMP agarose solution to $45^{\circ} \mathrm{C}$ in the water bath and hold there in preparation for lung inflation.

d. Chill Heparin $/ \mathrm{NaCl}$ solution on ice. 
Please cite this article as: Steffes, L. C. and Kumar, M. E. (2022). Chronic Daily House Dust Mite Exposure in Mice is an Effective Model to Quantify the Effect of Pharmacologic Agents on Discrete Stages of Artery Remodeling in Pulmonary Hypertension. Bio-protocol 12(01): e4273. DOI:

e. Prepare $4 \%$ PFA solution, aliquot into $50 \mathrm{~mL}$ conical tubes ( $40 \mathrm{~mL} \mathrm{4 \%} \mathrm{PFA} \mathrm{per} \mathrm{tube),} \mathrm{and}$ chill on ice. $40 \mathrm{~mL}$ is needed per heart-lung block collected.

f. Prepare collection tubes needed for any planned preparations not fixed with 4\% PFA:

i. Paraffin sections: Aliquot $10 \mathrm{~mL}$ of $10 \%$ neutral buffered formalin into $15 \mathrm{~mL}$ conical tubes and chill on ice. Per lobe collected, $10 \mathrm{~mL}$ is needed.

ii. RNA or protein collection: Sterile nuclease-free $1.5 \mathrm{~mL}$ tube, chilled on ice. One tube is needed per lobe collected. Follow an appropriate RNA or protein isolation protocol for whatever downstream experiments are planned.

g. Cover Styrofoam dissection board with fresh aluminum foil and secure foil with lab tape.

h. Assemble dissection tools and supplies in easy reach of dissection station: Stack of c-folded paper towels, $70 \%$ ethanol in spray bottle, sharp and blunt curved forceps, dissection scissors, butterfly needle attached to $50 \mathrm{~mL}$ syringe, blunt cannula attached to $5 \mathrm{~mL}$ syringe, suture thread, ice bucket with aliquoted fixatives, and a lab marker for labeling tubes.

2. Dissection for visualization of heart, lungs, and trachea

a. Following hemodynamic assessment, euthanize animals by severing the descending aorta while still under isoflurane anesthesia.

Tip: If hemodynamic measurement is not required, euthanize animals by carbon dioxide administration (or whatever approved method is preferred). Since inhaled anesthetics, $100 \%$ oxygen, and carbon dioxide alter vascular tone, for quantitative assessment of vessel remodeling parameters (detailed in "Vessel Remodeling Quantification", below) all animals to be compared should be euthanized in the same manner.

b. Place the euthanized mouse on several stacked paper towels on a dissecting board, ventral side up, with limbs extending laterally and secured with pins.

c. Spray the ventral surface with a spray bottle filled with $70 \%$ ethanol to wet the fur and decrease shedding into the thoracic cavity during dissection.

d. Lift the skin below the ribs with blunt serrated forceps and make a horizontal cut through the skin with dissection scissors, revealing the peritoneum.

e. Make a vertical cut through the skin from the original incision to the sternum and pull the skin to the sides, to reveal the chest and anterior abdomen.

f. Lift the peritoneum with blunt serrated forceps and again cut horizontally, allowing visualization of the abdominal organs.

g. Lift the liver and bisect the hepatic artery and portal vein, to serve as outflow during lung perfusion.

h. Grasp the xyphoid process, lift with forceps, and make a shallow incision with dissection scissors in the diaphragm (take care to avoid inadvertent laceration of the lungs). Allow the lungs to collapse with release of negative intrathoracic pressure.

i. Cut the ventral rib cage approximately $0.5 \mathrm{~cm}$ lateral to the sternum on both sides, to allow full visualization of the heart and the lungs (Figure $5 \mathrm{~A}$ ). 
Please cite this article as: Steffes, L. C. and Kumar, M. E. (2022). Chronic Daily House Dust Mite Exposure in Mice is an Effective Model to Quantify the Effect of Pharmacologic Agents on Discrete Stages of Artery Remodeling in Pulmonary Hypertension. Bio-protocol 12(01): e4273. DOI:

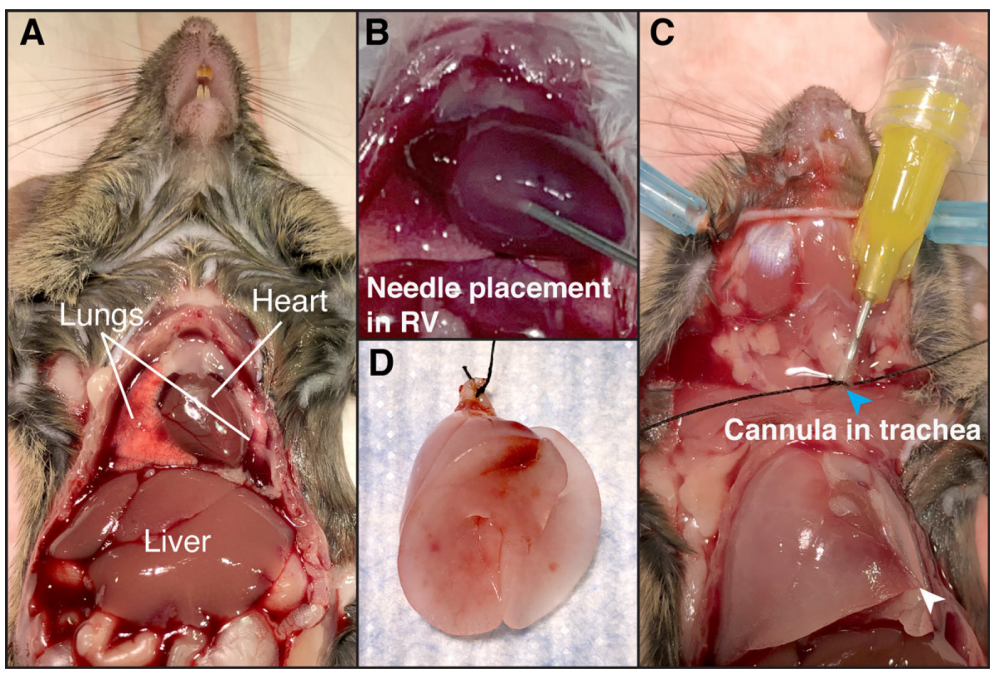

Figure 5. Lung perfusion and inflation.

Following hemodynamic assessment and euthanasia, the chest is opened to reveal the heart and lungs $(A)$, and saline is slowly injected into the right ventricle (RV) of the heart (B) to remove blood from the pulmonary circulation. To inflate the lungs with LMP agarose, the skin of the ventral throat is dissected open to reveal the trachea. A small cut is made in the trachea, an LMP agarose-filled syringe fitted with a blunt cannula is inserted (C), and suture thread is tightened around the trachea and cannula to make a seal (blue arrowhead in C). Inflation is considered complete when the lobe tips are rigid (white arrowhead in C). D, Heart-lung block removed from the chest following perfusion and agarose inflation.

3. Lung perfusion

a. Draw up $20 \mathrm{~mL}$ of Heparin/ $\mathrm{NaCl}$ solution into a $50 \mathrm{~mL}$ syringe, attach a 27 -gauge butterfly needle, and flush air from tubing.

b. Insert the needle into the right ventricle of the heart (Figure 5B) and perfuse at $<100 \mu \mathrm{L}$ per second, to wash blood from the pulmonary vascular bed for 2-3 min.

Tip: In healthy animals, the lungs will turn completely white as blood cells are flushed from the pulmonary circulation; perfusion will be notably less effective in animals with remodeling in the pulmonary arteries.

4. Lung inflation

a. Using blunt forceps and dissection scissors, make a vertical cut through the ventral skin of the neck, revealing the trachea.

b. Cut a 4-inch piece of suture thread, lace under the trachea with sharp-tipped curved forceps at the level of the manubrium, and tie into a loose surgical knot (to be tightened later around the intra-tracheal cannula).

c. Make a small incision in the ventral trachea above the level of the knot, just wide enough to allow for the insertion of the cannula. 
Please cite this article as: Steffes, L. C. and Kumar, M. E. (2022). Chronic Daily House Dust Mite Exposure in Mice is an Effective Model to Quantify the Effect of Pharmacologic Agents on Discrete Stages of Artery Remodeling in Pulmonary Hypertension. Bio-protocol 12(01): e4273. DOI:

Tip: If RNA or protein are to be prepared from individual lobes (see "Tissue fixation and handling", below, and Figure 6), ligate the primary bronchus leading to those lobes with suture thread prior to agarose inflation.

d. Remove the bottle of $2 \% \mathrm{LMP}$ agarose from the $45^{\circ} \mathrm{C}$ water bath, draw $3 \mathrm{~mL}$ of solution into a $5 \mathrm{~mL}$ syringe fitted with a 20-gauge blunt tip cannula.

Tip: Work quickly so agarose does not have time to polymerize inside the cannula.

e. Insert the cannula into the tracheal incision and advance down the trachea, until the cannula tip is approximately $3 \mathrm{~mm}$ above the level of the carina.

Tips:

i) Confirm that the $2 \%$ LMP agarose solution is still liquid by squirting out a small amount from the tip of the cannula prior to inserting it in the trachea. Once the suture is tightened around the intra-tracheal cannula, replacing the cannula is difficult.

ii) If LMP agarose solution routinely polymerizes before it can be used for inflation, 1) raise the temperature of the water bath very slightly $\left(\sim 2^{\circ} \mathrm{C}\right)$, or 2) squirt out a small volume from the filled cannula onto a paper towel every $\sim 10 \mathrm{~s}$, to eject cooled agarose from the cannula tip and replace it with warm agarose from the syringe chamber.

f. Tighten the surgical knot, making a firm seal between the trachea and the cannula, and gently inject molten LMP agarose into the trachea to inflate the lungs (Figure 5C).

g. Monitor lung inflation carefully, and stop when the distal tip of the accessory lobe is rigid (Figure 5C-5D).

Tip: Take care not to over-inflate the lungs, which will damage parenchymal morphology.

h. Remove the cannula and immediately tighten the tracheal ligature to prevent outflow of agarose.

Tip: Cannulae can be reused several times if they are immediately flushed with sterile PBS or water, to remove residual LMP agarose.

i. Wait until the LMP agarose has solidified, approximately 2-4 min, before continuing the dissection.

5. Removal of heart-lung block

a. With dissecting scissors cut and remove the ventral clavicle.

b. Using blunt serrated forceps, grasp the esophagus just above the level of the liver, and pull gently upward, lifting the heart-lung block from the chest cavity.

c. Clip and dissect away any adhesions binding the heart and lung tissue to the dorsal chest wall up to the level of the trachea.

d. Cut the trachea above the ligature and rinse the heart-lung block briefly in cold PBS in a Petri dish on ice.

6. Tissue fixation and handling

Note: Multiple tissue preparations can be prepared from the heart-lung block of each animal, if desired. A possible example of how a heart-lung block can be divided between multiple preparations is shown in Figure 6. In addition to reserving a lobe for recovery of bulk RNA or 
Please cite this article as: Steffes, L. C. and Kumar, M. E. (2022). Chronic Daily House Dust Mite Exposure in Mice is an Effective Model to Quantify the Effect of Pharmacologic Agents on Discrete Stages of Artery Remodeling in Pulmonary Hypertension. Bio-protocol 12(01): e4273. DOI:

protein (see "Lung inflation", above), it is practical to prepare paraffin sections, cryosections, vibratome sections, and dehydrate the remaining tissue in methanol for long term storage at $20^{\circ} \mathrm{C}$. Both fresh and methanol-dehydrated hearts can be dissected to calculate the Fulton Index (Ma et al., 2016). We have never observed differences in the rate or extent of remodeling between different lung lobes within the same animal, and find that dividing the lung between multiple preparations allows more efficient use of precious animals.

a. If paraffin sections are required, remove one lobe prior to fixation and immerse it in the prepared tube of $10 \%$ neutral buffered formalin for $48 \mathrm{~h}$ at RT. Then, transfer it to a biopsy tissue cassette, wash in $70 \%$ ethanol, paraffin embed, and section following standard protocols.

Tip: This tissue is suitable for histological stains (H\&E, Movat's pentachrome, etc.), immunohistochemistry (Hofman and Taylor, 2013), or RNA in situ hybridization protocols, including RNAscope (Wang et al., 2012).

b. Immerse all remaining portions of the heart-lung block in the prepared tube of ice cold $4 \%$ PFA and fix for $4 \mathrm{~h}$ with gentle shaking at $4^{\circ} \mathrm{C}$.

c. After PFA fixation, divide the lung into individual lobes for the desired staining preparations:

i. Prepare lobes for cryosectioning by immersing them in $30 \%$ sucrose in PBS $(10 \mathrm{~mL}$ per lobe in a $15 \mathrm{~mL}$ Falcon tube). Incubate overnight at $4^{\circ} \mathrm{C}$ with gentle shaking.

ii. Transfer lobes for vibratome sectioning into cold PBS, store at $4^{\circ} \mathrm{C}$, and section within $24 \mathrm{~h}$. Further information on whole mount staining and deep tissue imaging of vibratome sections can be found in Steffes et al. (2020) and Gillich et al. (2020).

iii. Dehydrate the remaining lobes, trachea, and heart through a methanol series $(25 \%$, $50 \%$, and $75 \%$ methanol in $\mathrm{diH}_{2} \mathrm{O}, 30$ min each), and transfer to $100 \%$ methanol for long term storage at $-20^{\circ} \mathrm{C}$ in well-sealed $50 \mathrm{~mL}$ conical tubes. Lobes stored in methanol can be rehydrated and sectioned as needed, or stained as whole mount preparations (Metzger et al., 2008).

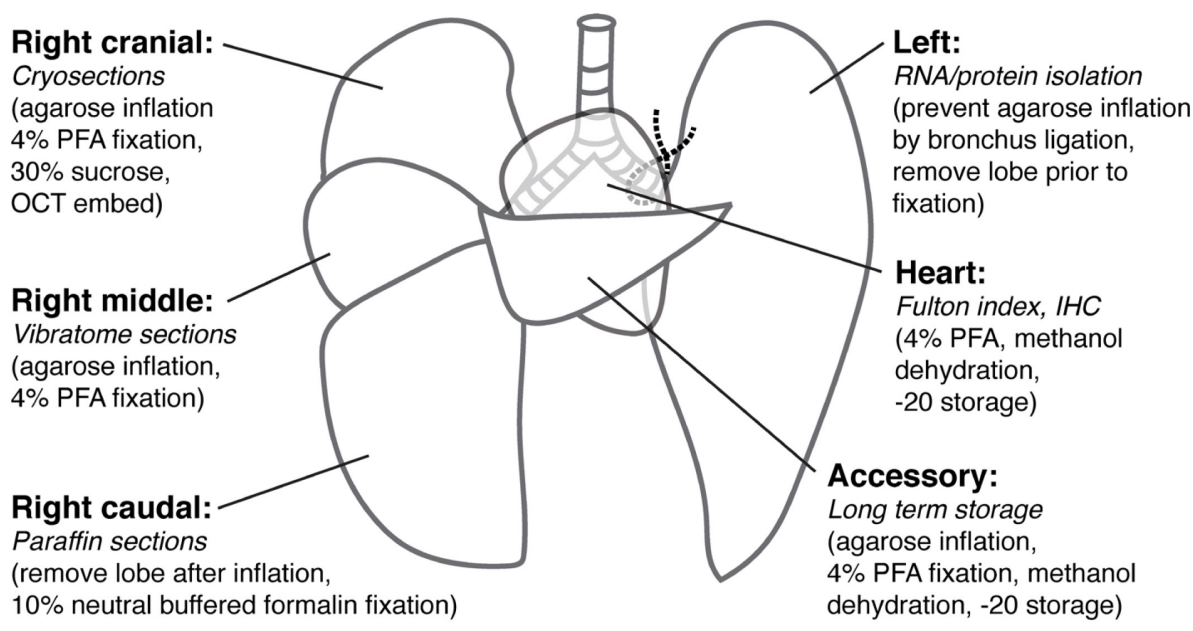

Figure 6. Multiple tissue preparations can be prepared from the heart-lung block of a single animal. 
Please cite this article as: Steffes, L. C. and Kumar, M. E. (2022). Chronic Daily House Dust Mite Exposure in Mice is an Effective Model to Quantify the Effect of Pharmacologic Agents on Discrete Stages of Artery Remodeling in Pulmonary Hypertension. Bio-protocol 12(01): e4273. DOI:

After perfusion, airways of individual lobes can be ligated prior to agarose inflation for RNA or protein extraction (Left lobe; ligation indicated with dotted line). Lobes intended for paraffin sections are removed after inflation and fixed with 10\% neutral buffered formalin (Right caudal lobe). All other preparations (cryosections, vibratome sections, long term storage in methanol, etc.) are completed on tissue fixed with $4 \%$ paraformaldehyde and subsequently divided for individual treatments. Schematic illustrates one possible plan for dividing a heart-lung block between six different preparations. We have observed no difference in the degree of pulmonary arterial remodeling between different lobes of the lung from the same mouse.

F. Cryosections and immunohistochemistry panel to highlight vessel compartments relevant for quantitation

1. Remove lobes from sucrose, blot briefly on paper towels.

2. Position lobes in OCT-filled cryomolds and cover lung completely with OCT.

3. Freeze lungs in cryomolds on a bed of dry ice.

4. Store blocks in air-tight bags at $-80^{\circ} \mathrm{C}$ until ready to section.

5. Cut $20 \mu \mathrm{m}$ sections using a cryostat.

6. Collect the sections on Superfrost + glass microscope slides.

7. Air dry for $10 \mathrm{~min}$, and store in slide boxes at $-80^{\circ} \mathrm{C}$.

8. To stain, thaw individual slides on the lab bench and allow condensation to fully evaporate.

9. Draw boundary around sections with a hydrophobic pen.

10. Remove OCT with two 5-min washes in PBST.

Tip: We find that damage to sections is minimized by using a "pool and tip method" (Figure 7) for solution incubation and washing.

a. Pool solution directly onto horizontal slides with a disposable transfer pipette (Figure 7A).

b. Incubate at RT in a humid chamber (Figure 7C) on lab bench without coverslipping for the indicated length of time.

c. Remove solutions by tipping the liquid off slide onto a dry paper towel without touching the slide surface (Figure 7B). 
Please cite this article as: Steffes, L. C. and Kumar, M. E. (2022). Chronic Daily House Dust Mite Exposure in Mice is an Effective Model to Quantify the Effect of Pharmacologic Agents on Discrete Stages of Artery Remodeling in Pulmonary Hypertension. Bio-protocol 12(01): e4273. DOI: 10.21769/BioProtoc.4273.

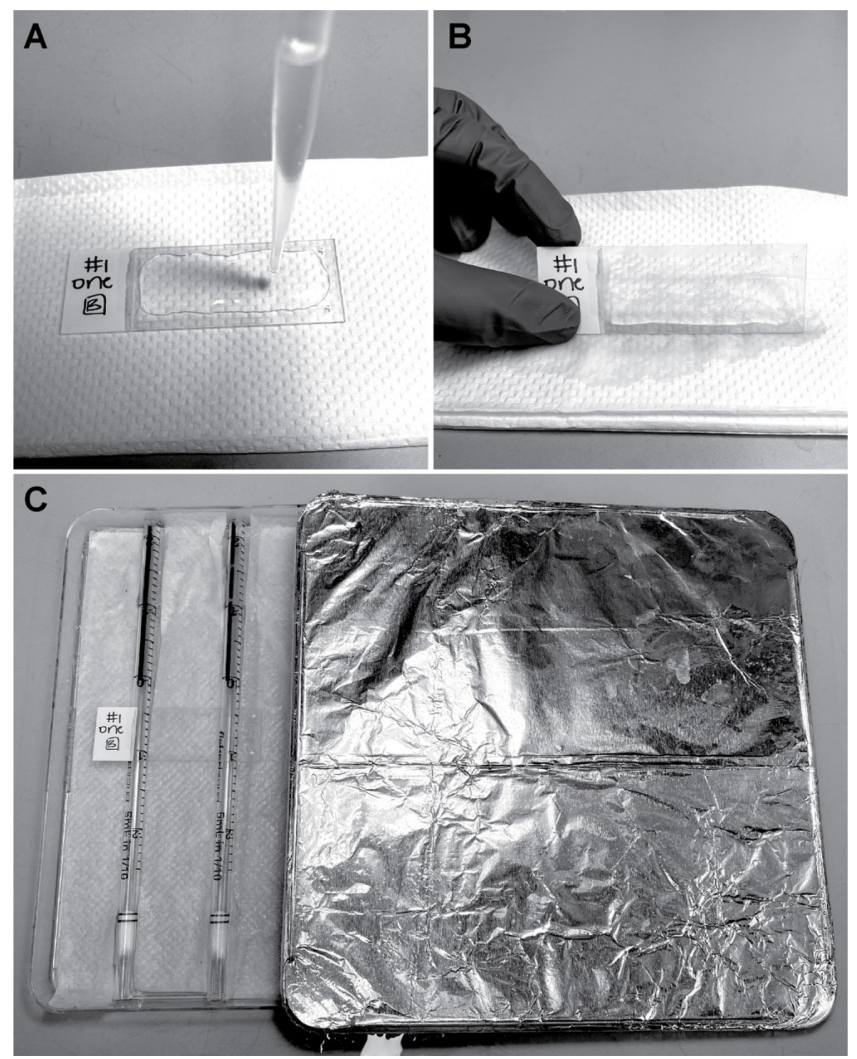

Figure 7. Pool and tip staining method minimizes damage to sections.

A. Draw barrier around tissue sections with hydrophobic pen and gently pool solutions directly on horizontal slides without coverslipping. B. Remove solution after required incubation period by tipping onto a clean paper towel. C. A homemade humid chamber - made from a $241 \times 241$ $\times 20 \mathrm{~mm}$ square bioassay dish with $5 \mathrm{~mL}$ disposable pipettes cut to length as slide rests, watersaturated paper towel cut to size to keep the atmosphere moist, and covered with foil to protect from the light - holds up to 14 slides. Staining is effective even without large volume washes or agitating slides during washes, and section loss or damage is minimal.

11. To block sections, pool $\sim 300 \mu \mathrm{L}$ of freshly made preblock solution for $30 \mathrm{~min}-4 \mathrm{~h}$ at RT in a humid chamber

Tip: Serum species should be adjusted to match the species in which the secondary antibodies have been raised.

12. Remove preblock via tip method and apply the primary antibody solution diluted in preblock pooled on horizontal slide.

13. Incubate overnight in a humid chamber at RT protected from light.

Tip: A simple staining panel is shown in Table 1 that allows visualization of multiple relevant vessel compartments important during vascular remodeling and facilitates remodeling quantitation measurements (see Figure 2B).

14. Wash slides with PBST three times for 5 min each, using the pool and tip method.

15. Apply secondary antibody solution diluted in preblock pooled on horizontal slide. 
Please cite this article as: Steffes, L. C. and Kumar, M. E. (2022). Chronic Daily House Dust Mite Exposure in Mice is an Effective Model to Quantify the Effect of Pharmacologic Agents on Discrete Stages of Artery Remodeling in Pulmonary Hypertension. Bio-protocol 12(01): e4273. DOI:

16. Incubate for $45 \mathrm{~min}$ at $\mathrm{RT}$ in a humid chamber protected from light.

17. Wash slides with PBST three times for 5 min each using the pool and tip method.

18. Coverslip with a firm-setting aqueous mounting medium (e.g., ProlongGold) and $60 \mathrm{~mm}$ coverslips.

Tips:

i) Allow at least $2 \mathrm{~h}$ for the mounting medium to polymerize prior to imaging to avoid damaging the tissue sections.

ii) Image slides within $48 \mathrm{~h}$ of coverslipping for optimal image quality.

Table 1. Staining panel to highlight major vessel compartments and image remodeling.

\begin{tabular}{|c|c|c|c|c|c|c|}
\hline $\begin{array}{l}\text { Channel } \\
\text { (laser) }\end{array}$ & $\begin{array}{l}\text { Primary Mix } \\
\text { (highlighted structure } \\
\text { or cell type) }\end{array}$ & Dilution & Ordering Information & $\begin{array}{l}\text { Secondary } \\
\text { Mix }\end{array}$ & Dilution & $\begin{array}{l}\text { Ordering } \\
\text { information }\end{array}$ \\
\hline Blue $(405 \mathrm{~nm})$ & DAPI (cell nuclei) & $1: 1,000$ & Invitrogen, D1306 & (no secondary) & & \\
\hline Green $(488 \mathrm{~nm})$ & $\begin{array}{ll}\text { Hydrazide } & \text { A488 } \\
\text { (elastin) } & \end{array}$ & $1: 200$ & $\begin{array}{l}\text { Invitrogen, } \mathrm{A} 10436 \\
\text { (dissolve in } 2 \mathrm{~mL} \text { of } \\
\mathrm{dH}_{2} \mathrm{O} \text { to make a } 0.5 \\
\mathrm{mg} / \mathrm{mL} \text { stock) }\end{array}$ & (no secondary) & & \\
\hline Red $(561 \mathrm{~nm})$ & $\begin{array}{l}\text { Mouse a-SMA Сy3 } \\
\text { (SMCs \& neointima) }\end{array}$ & $1: 200$ & Sigma, C6198 & (no secondary) & & \\
\hline Far red $(633 \mathrm{~nm})$ & $\begin{array}{l}\text { Armenian Hamster } \alpha- \\
\text { CD31 (endothelium) }\end{array}$ & $1: 200$ & Bio-Rad, MCA1370Z & $\begin{array}{l}\text { Goat } \quad \alpha- \\
\text { Armenian } \\
\text { Hamster } \text { A647 }\end{array}$ & $1: 250$ & $\begin{array}{l}\text { Invitrogen, } \\
\text { A-21451 }\end{array}$ \\
\hline
\end{tabular}

G. Vessel Remodeling Quantification

1. Blind the researchers carrying out image collection and quantitation by having another lab member obscure the slide labels with opaque tape coded with random identifiers.

2. Using a confocal microscope, scan lung sections at low magnification to identify arteries between $20 \mu \mathrm{m}$ and $200 \mu \mathrm{m}$ in diameter for further inspection.

Tip: Cross-sectional views are preferred as they simplify quantitation. Longitudinal cuts can be quantitated, if necessary, but significant tissue thickness (or access to serial sections) is required to identify the true center plane of the artery.

3. Increase magnification (to $25-40 \times$ ) once an appropriate artery cross section is located

4. Collect a high-resolution image containing the most comprehensive view of the medial smooth muscle layer, neointimal lesion and endothelial cells.

5. Collect images of all arteries within the $20-200 \mu \mathrm{m}$ size range found within each section without selection for degree of remodeling, position in the lobe, etc.

Tip: Arteries can be distinguished from veins by both location and vessel anatomy (Figure 8):

i) Arteries are found with an accompanying airway, and contain both inner and outer elastic laminae, which will be marked by hydrazide (Figure 8A-8B).

ii) Veins are located far from airways, and have a single elastic lamina (Figure $8 A-8 C$ ). 
Please cite this article as: Steffes, L. C. and Kumar, M. E. (2022). Chronic Daily House Dust Mite Exposure in Mice is an Effective Model to Quantify the Effect of Pharmacologic Agents on Discrete Stages of Artery Remodeling in Pulmonary Hypertension. Bio-protocol 12(01): e4273. DOI:

6. Using image analysis software (Zen Blue, Zen Black, or ImageJ) obtain two orthogonal sets of measurements for each vessel (Figure 2B). Measurements should include:

a. External vessel diameter (outside margin of external elastic laminae).

b. Media thickness (inside margin of external elastic lamina to outside margin of internal elastic lamina). There will be two media thickness measurements for each of the two orthogonal measurement axes. Media thickness is reported as an absolute measurement $(\mu \mathrm{m})$.

c. Neointima thickness (inside margin of internal elastic lamina to the edge of the endothelium). There will be two neointima thickness measurements for each of the two orthogonal measurement axes. Neointimal thickness can be reported as an absolute measurement, or a percentage of vessel diameter: sum the two neointima measurements for each measurement axis, divide by vessel diameter, and multiply by 100 .

7. Collect measurements from at least 50 arteries per mouse, to assure a broad survey of arteries, and allow for meaningful statistical analysis.

8. Record measurements into a spreadsheet (GoogleSheets or Excel).

9. Unblind the samples after measurements have been recorded from all samples, and add mouse number, drug treatment group, and length of HDM exposure for each measurement to the spreadsheet, to facilitate downstream statistical analysis.
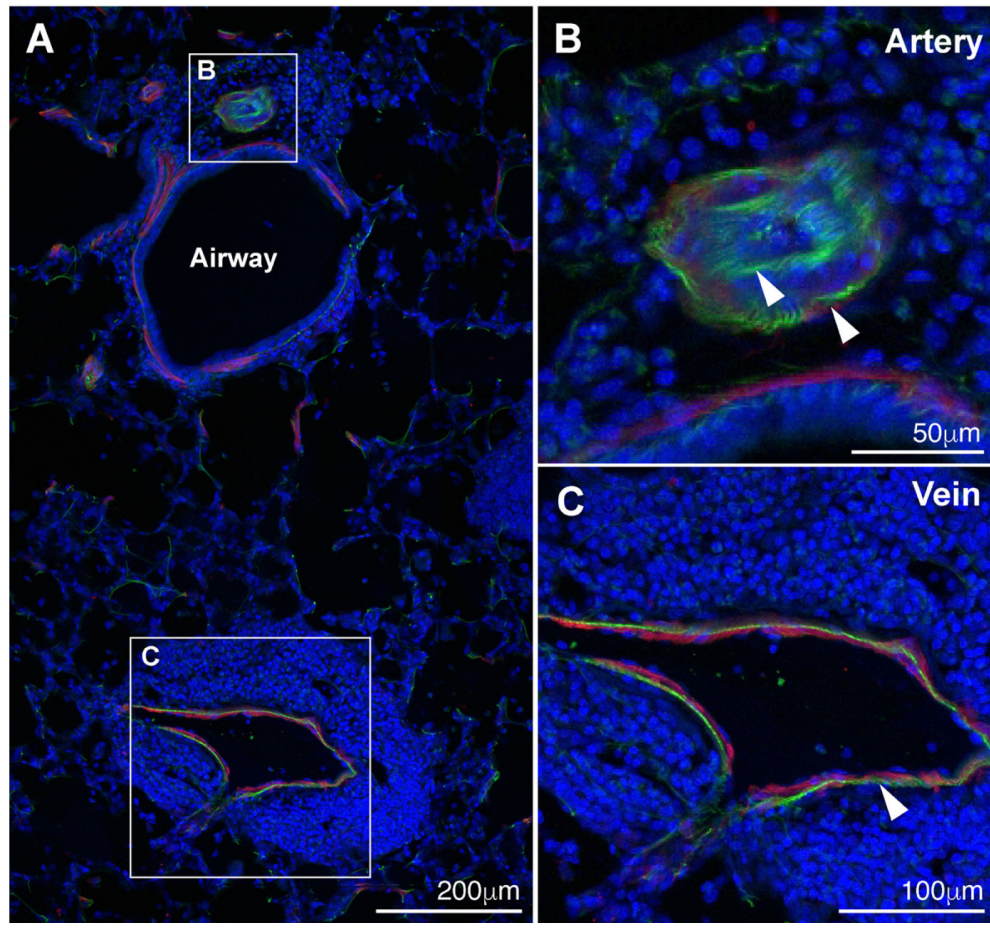

Figure 8. Arteries and veins can be distinguished by location and elastin staining.

A. Arteries run close to airways while veins are positioned far from airways. B. Arteries have both an internal and external elastic lamina, while veins (C) have only a single elastin layer. Elastin visualized with hydrazide staining (green); smooth muscle cells highlighted by SMA antibody staining (red); nuclei stained with DAPI (blue). Arrowheads, elastic laminae. 
Please cite this article as: Steffes, L. C. and Kumar, M. E. (2022). Chronic Daily House Dust Mite Exposure in Mice is an Effective Model to Quantify the Effect of Pharmacologic Agents on Discrete Stages of Artery Remodeling in Pulmonary Hypertension. Bio-protocol 12(01): e4273. DOI:

\section{Data analysis}

Detailed discussion of data analysis can be found in the original research paper associated with this protocol (Steffes et al., 2020). Statistical analysis can be performed in either Excel or the statistical language $\mathrm{R}$ using $\mathrm{R}$ Studio as we have done here.

1. Compare PBS-exposed animals to each collected timepoint in the HDM time course (e.g., PBS vs. 2 weeks HDM, etc.) using standard ANOVA analysis. This will identify which remodeling events (medial thickening, neointima establishment, or neointimal lesion growth) change significantly during each time window studied.

2. Identify any changes between drug- and vehicle-exposed animals at each time point, using twoway ANOVA analysis.

3. Create scatter plots showing the per-vessel quantitation of medial thickness and neointimal lesion thickness, with overlayed box and whisker plots, and p-values in R, using the plotting package ggplot2 (or equivalent plotting software), as we have done in Figure $2 \mathrm{C}$.

\section{$\underline{\text { Recipes }}$}

1. $1 \times$ Phosphate buffered saline (PBS)

Dilute $100 \mathrm{~mL}$ of $10 \times$ PBS with $900 \mathrm{~mL}$ of sterile $\mathrm{dH}_{2} \mathrm{O}$

2. House Dust Mite Solution (HDM)

Dilute lyophilized house dust mite extract to $50 \mu \mathrm{g}$ Derp1 protein/120 $\mu \mathrm{L}$ of PBS

3. Heparin/ $\mathrm{NaCl}$

Dissolve $9 \mathrm{~g} \mathrm{NaCl}$ in $1 \mathrm{~L}$ of PBS, then add $56 \mathrm{mg}$ Heparin

4. $2 \%$ low melting point (LMP) agarose

Add $4 \mathrm{~g}$ of LMP agarose to $200 \mathrm{~mL}$ of PBS. Boil in microwave until the solution is clear

5. $4 \%$ paraformaldehyde

Dissolve $10 \mathrm{~mL}$ of $16 \%$ PFA in $30 \mathrm{~mL}$ of PBS

6. $30 \%$ sucrose in PBS

Add $150 \mathrm{~g}$ sucrose to $500 \mathrm{~mL}$ of PBS, heat in microwave until dissolved. Cool to $4^{\circ} \mathrm{C}$ before applying to tissue

7. Methanol series

Dilute $100 \%$ methanol for staged tissue dehydration $\left(25 \%, 50 \%, 75 \%\right.$ methanol in $\left.\mathrm{dH}_{2} \mathrm{O}\right)$

8. PBST

$0.1 \%$ Tween 20 in PBS

Dissolve $0.5 \mathrm{~mL}$ of Tween 20 in $500 \mathrm{~mL}$ of PBS.

9. Preblock

$0.3 \%$ Triton $\mathrm{X}-100$

$5 \%$ goat serum

$1.5 \%$ BSA in PBS 
Please cite this article as: Steffes, L. C. and Kumar, M. E. (2022). Chronic Daily House Dust Mite Exposure in Mice is an Effective Model to Quantify the Effect of Pharmacologic Agents on Discrete Stages of Artery Remodeling in Pulmonary Hypertension. Bio-protocol 12(01): e4273. DOI:

Dilute Triton X-100 to $10 \%$ stock solution by addition $1 \mathrm{~mL}$ of Triton X-100 to $9 \mathrm{~mL}$ of PBS. For $10 \mathrm{~mL}$ preblock: in $15 \mathrm{~mL}$ conical tube, add $300 \mu \mathrm{L}$ of $10 \%$ Triton, $500 \mu \mathrm{L}$ of serum, $150 \mathrm{mg}$ Bovine Serum Albumin (BSA), and bring volume to $10 \mathrm{~mL}$ with PBS.

\section{Acknowledgments}

This work was supported by Stanford University Department of Pediatrics Bridge to K Program and the Francis Family Foundation, Parker B. Francis Fellowship to L.C.S., and American Heart Association 16SDG30030006, Stanford Spectrum-Child Health Research Institute seed grants, Bravo Family endowed faculty scholarship and Vera Moulton Wall Center for Pulmonary Vascular Disease research grants to M.E.K. The original research paper from which this protocol derives is Steffes et al. (2020).

\section{Competing interests}

None of the authors have financial or non-financial competing interests.

\section{Ethics}

All animal experiments received IRB approval from the Stanford University Institutional Animal Care and Use Committee (APLAC 31869, first approved September 6, 2016 and valid through September 23, 2022.

\section{References}

1. Dai, Z., Li, M., Wharton, J., Zhu, M. M. and Zhao, Y. Y. (2016). Prolyl-4 Hydroxylase 2 (PHD2) Deficiency in Endothelial Cells and Hematopoietic Cells Induces Obliterative Vascular Remodeling and Severe Pulmonary Arterial Hypertension in Mice and Humans Through Hypoxia-Inducible Factor-2a. Circulation 133(24): 2447-2458.

2. Gillich, A., Zhang, F., Farmer, C. G., Travaglini, K. J., Tan, S. Y., Gu, M., Zhou, B., Feinstein, J. A., Krasnow, M. A. and Metzger, R. J. (2020). Capillary cell-type specialization in the alveolus. Nature 586(7831): 785-789.

3. Gomez-Arroyo, J., Saleem, S. J., Mizuno, S., Syed, A. A., Bogaard, H. J., Abbate, A., Taraseviciene-Stewart, L., Sung, Y., Kraskauskas, D., Farkas, D., et al. (2012). A brief overview of mouse models of pulmonary arterial hypertension: problems and prospects. Am J Physiol Lung Cell Mol Physiol 302(10): L977-991.

4. Hofman, F. M. and Taylor, C. R. (2013). Immunohistochemistry. Curr Protoc Immunol 103: 21 24 21-21 2426. 
Please cite this article as: Steffes, L. C. and Kumar, M. E. (2022). Chronic Daily House Dust Mite Exposure in Mice is an Effective Model to Quantify the Effect of Pharmacologic Agents on Discrete Stages of Artery Remodeling in Pulmonary Hypertension. Bio-protocol 12(01): e4273. DOI:

5. Humbert, M., Guignabert, C., Bonnet, S., Dorfmuller, P., Klinger, J. R., Nicolls, M. R., Olschewski, A. J., Pullamsetti, S. S., Schermuly, R. T., Stenmark, K. R., et al. (2019). Pathology and pathobiology of pulmonary hypertension: state of the art and research perspectives. Eur Respir J 53(1).

6. Lau, E. M. T., Giannoulatou, E., Celermajer, D. S. and Humbert, M. (2017). Epidemiology and treatment of pulmonary arterial hypertension. Nat Rev Cardiol 14(10): 603-614.

7. Ma, Z., Mao, L. and Rajagopal, S. (2016). Hemodynamic Characterization of Rodent Models of Pulmonary Arterial Hypertension. J Vis Exp(110): 53335.

8. Metzger, R. J., Klein, O. D., Martin, G. R. and Krasnow, M. A. (2008). The branching programme of mouse lung development. Nature 453(7196): 745-750.

9. Simonneau, G., Montani, D., Celermajer, D. S., Denton, C. P., Gatzoulis, M. A., Krowka, M., Williams, P. G. and Souza, R. (2019). Haemodynamic definitions and updated clinical classification of pulmonary hypertension. Eur Respir J 53(1).

10. Spiekerkoetter, E., Kawut, S. M. and de Jesus Perez, V. A. (2019). New and Emerging Therapies for Pulmonary Arterial Hypertension. Annu Rev Med 70: 45-59.

11. Steffes, L. C., Froistad, A. A., Andruska, A., Boehm, M., McGlynn, M., Zhang, F., Zhang, W., Hou, D., Tian, X., Miquerol, L., et al. (2020). A Notch3-Marked Subpopulation of Vascular Smooth Muscle Cells Is the Cell of Origin for Occlusive Pulmonary Vascular Lesions. Circulation 142(16): 1545-1561.

12. Stenmark, K. R., Fagan, K. A. and Frid, M. G. (2006). Hypoxia-induced pulmonary vascular remodeling: cellular and molecular mechanisms. Circ Res 99(7): 675-691.

13. Wang, F., Flanagan, J., Su, N., Wang, L. C., Bui, S., Nielson, A., Wu, X., Vo, H. T., Ma, X. J. and Luo, Y. (2012). RNAscope: a novel in situ RNA analysis platform for formalin-fixed, paraffinembedded tissues. J Mol Diagn 14(1): 22-29. 